\title{
Conjugate natural convection in a square enclosure under horizontal magnetic field effect
}

\author{
Abdennacer Belazizia ${ }^{1,2,3, a}$, Said Abboudi ${ }^{3}$ And Smail BenissaAd ${ }^{1,2}$ \\ 1 Université Kasdi Merbah, Ouargla, Algérie \\ 2 Laboratoire d'Energétique Appliquée et de la Pollution, Université Constantine 1, Algérie \\ 3 Institut IRTES, Laboratoire M3M, site de Sévenans, UTBM, 90000 Belfort Cedex, France
}

Received 19 April 2014, Accepted 9 January 2015

\begin{abstract}
Steady, laminar, conjugate natural convection flow in a square enclosure is considered. Both effects: conduction in a vertical wall and the presence of a magnetic field are taken together. The enclosure is filled with liquid gallium and subjected to horizontal temperature gradient. The main focus of the study is examining the effect of Hartmann number on fluid flow and heat transfer. The effect of Rayleigh number and conduction in the left wall is also considered. The obtained result show in the absence of a magnetic field that natural convection can be strengthed by the increase of both Rayleigh number and conductivity ratio, because of the increase of the effective temperature difference driving the flow. For poor conducting wall, where the solid part is an insulated material and the thermal resistance is more important the average Nusselt number is approximately constant and having low values comparing with equal and high conducting wall, indicating that most of heat transfer is by heat conduction. In the presence of a magnetic field the results show that for a given Rayleigh number, as the value of Hartmann number increases, convection is suppressed progressively and the rate of heat transfer is reduced in the enclosure.
\end{abstract}

Key words: Conjugate natural convection / magnetic field / electrically conducting fluid / Square enclosure / finit volume method

\section{Introduction}

Natural convection in enclosures is a topic of considerable engineering interest. Applications range from thermal design of buildings, to cryogenic storage, furnace design, nuclear reactor design, and others. The problem of natural convection flow in a square and rectangular enclosure with uniform temperature at vertical walls and insulated top and bottom walls has been the subject of many studies. The walls of the enclosure are assumed to be of zero thickness and conduction is not accounted for this. However, in many practical situations, especially those concerned with the design of thermal insulation, conduction in the walls can have an important effect on the natural convection flow in the enclosure [1-3].

In addition the process of manufacturing materials in industrial problems (for example, crystal growth using the horizontal Bridgman technique) involves an electrically conducting fluid subjected to a magnetic field (MHD

\footnotetext{
${ }^{a}$ Corresponding author: nacer.belazizia@gmail.com
}

convection). In that case the fluid experiences an electromagnetic force (Lorentz force) and its effect is to control natural convection by reducing the flow velocity. This in turn affects the rate of heat and mass transfer [4-6].

According to the above studies, we conclude that fluid flow and heat transfer in enclosures are strongly influenced by both effects: the presence of magnetic field and thermal conduction in walls presenting a thickness.

In most of the studies presented in literature on natural convection in differentially heated cavity has not been considered the above said effects together. The present work aims to study the effect of wall thickness in a square enclosure on natural convection of conducting fluid in the presence of magnetic field. The cavity is filled with liquid gallium $(P r=0.02)$ and submitted to horizontal temperature gradient. The applied magnetic field is assumed to be in the horizontal direction. The main focus is on examining the effect of Rayleigh number and conduction in the wall, on fluid motion flow and heat transfer. The effect of the magnetic field strength is also considered. 


\section{Nomenclature}

\begin{tabular}{|c|c|}
\hline $\bar{B}$ & Applied magnetic field $\mathrm{T}$ \\
\hline$D$ & Dimensionless wall thickness $D=d / H$ \\
\hline $\boldsymbol{F}_{\mathrm{EM}}$ & Lorentz force, N.m ${ }^{-3}$ \\
\hline$H$ & Wall height m \\
\hline $\mathrm{Ha}$ & Hartmann number \\
\hline$J$ & Current density, A.m ${ }^{-2}$ \\
\hline$K r$ & Thermal conductivity ratio $K r=k_{w} / k_{f}$ \\
\hline$k_{w}$ & Thermal conductivity of the solid wall w. $\mathrm{m}^{-1} \cdot \mathrm{K}^{-1}$ \\
\hline$k_{f}$ & Thermal conductivity of the fluid $\mathrm{w} \cdot \mathrm{m}^{-1} \cdot \mathrm{K}^{-1}$ \\
\hline$L$ & Cavity length $\mathrm{m}$ \\
\hline$N u$ & Local Nusselt number \\
\hline$\overline{N u}$ & Average Nusselt number \\
\hline $\operatorname{Pr}$ & Prandtl number of the fluid $(v / \alpha)$ \\
\hline$R a$ & Rayleigh number $g \beta H^{3}\left(T_{h}-T_{c}\right) / v \alpha$ \\
\hline$T$ & Temperature K \\
\hline$t^{*}$ & Time s \\
\hline & Dimensionless time $t^{*} /\left(H^{2} / \alpha\right)$ \\
\hline$u, v$ & Velocity components m.s ${ }^{-1}$ \\
\hline$U, V$ & Dimensionless velocity components $u /(\alpha / H), v /(\alpha / H)$ \\
\hline $\boldsymbol{V} \quad \mathrm{l}$ & Dimensionless velocity of the flow \\
\hline$p$ & Pressure N.m ${ }^{-2}$ \\
\hline & Dimensionless pressure $p-p_{0} /(\alpha / H)^{2}$ \\
\hline$X, Y]$ & Non-dimensional Cartesian coordinates, $x / H, y / H$ \\
\hline & $\begin{array}{l}\text { Greek symbols } \\
\end{array}$ \\
\hline$\alpha^{*}$ & Thermal diffusivity ratio $\alpha_{w} / \alpha_{f}$ \\
\hline$\beta$ & Thermal expansion coefficient $\mathrm{K}^{-1}$ \\
\hline$\theta$ & Non-dimensional temperature $\left(T-T_{c}\right) /\left(T_{h}-T_{c}\right)$ \\
\hline$v$ & Kinematic viscosity $\mathrm{m}^{2} \cdot \mathrm{s}^{-1}$ \\
\hline$\sigma$ & Electrical conductivity of the medium $\Omega^{-1} \cdot \mathrm{m}^{-1}$ \\
\hline$\psi$ & Dimensionless stream function \\
\hline & Dimensionless vorticity \\
\hline & Subscripts \\
\hline$c$ & Cold \\
\hline$f$ & Fluid \\
\hline$h$ & Hot \\
\hline & Wall \\
\hline & Wall/fluid interface \\
\hline
\end{tabular}

\section{Problem geometry and governing equations}

The geometry of the problem is shown in Figure 1. The flow is two-dimensional, laminar and incompressible. All fluid properties are constant. The electrically conducting fluid is considered to be Newtonian. Viscous dissipation, heat generation and radiation effects are neglected. It is also assumed that the induced electric current and Joule heating are neglected. The Boussinesq approximation is applied : $\rho(T)=\rho_{0}\left[1-\beta\left(T-T_{0}\right)\right]$. The uniform magnetic field $\boldsymbol{B}$ of constant magnitude $B_{0}$ is applied.

$$
\begin{aligned}
\boldsymbol{B} & =B_{x} \boldsymbol{e}_{x}+B_{y} \boldsymbol{e}_{y} \\
B_{0} & =\left(B_{x}^{2}+B_{y}^{2}\right)^{1 / 2}
\end{aligned}
$$

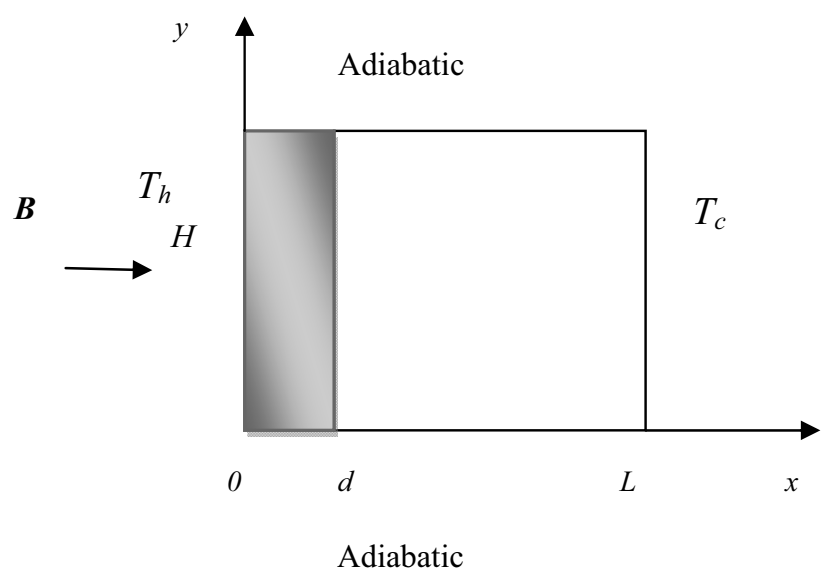

Fig. 1. Physical configuration.

where $\boldsymbol{e}_{x}$ and $\boldsymbol{e}_{y}$ are unit vectors in Cartesian coordinate system.

For a horizontal magnetic field $B_{y}=0$ and $B_{x}=B_{0}$.

The electric current $\boldsymbol{J}$ and the Lorentz force $\boldsymbol{F}_{\mathrm{EM}}$ are defined respectively by:

$$
\begin{aligned}
\boldsymbol{J} & =\sigma(\boldsymbol{V} \times \boldsymbol{B}) \\
\boldsymbol{F}_{\mathrm{EM}} & =\boldsymbol{J} \times \boldsymbol{B}=\sigma(\boldsymbol{V} \times \boldsymbol{B}) \times \boldsymbol{B}
\end{aligned}
$$

The Lorentz force compounds for horizontal magnetic field are:

$$
\left.\begin{array}{l}
F_{\mathrm{EM} x}=\sigma\left(v \cdot B_{x} \cdot B_{y}-u \cdot B_{y}^{2}\right)=0 \\
F_{\mathrm{EM} y}=\sigma\left(u \cdot B_{x} \cdot B_{y}-v \cdot B_{x}^{2}\right)=-\sigma v B_{0}^{2}
\end{array}\right\}
$$

The equations governing the conservation of mass, momentum and energy for an unsteady laminar flow in the presence of a horizontal magnetic field are:

- Fluid part

$$
\begin{aligned}
& \frac{\partial u}{\partial x}+\frac{\partial v}{\partial y} \\
& \frac{\partial u}{\partial t^{*}}+u \frac{\partial u}{\partial x}+v \frac{\partial u}{\partial y}=-\frac{1}{\rho} \frac{\partial p}{\partial x}+\nu\left(\frac{\partial^{2} u}{\partial x^{2}}+\frac{\partial^{2} u}{\partial y^{2}}\right) \\
& \frac{\partial v}{\partial t^{*}}+u \frac{\partial v}{\partial x}+v \frac{\partial v}{\partial y}=-\frac{1}{\rho} \frac{\partial p}{\partial y}+\nu\left(\frac{\partial^{2} v}{\partial x^{2}}+\frac{\partial^{2} v}{\partial y^{2}}\right) \\
& +g \beta\left(T_{f}-T_{0}\right)-\frac{\sigma v B^{2}}{\rho} \\
& \frac{\partial T_{f}}{\partial t^{*}}+u \frac{\partial T_{f}}{\partial x}+v \frac{\partial T_{f}}{\partial y}=\alpha_{f}\left(\frac{\partial^{2} T_{f}}{\partial x^{2}}+\frac{\partial^{2} T_{f}}{\partial y^{2}}\right)
\end{aligned}
$$

- Solid part

$$
\frac{\partial T_{w}}{\partial t^{*}}=\alpha_{w}\left(\frac{\partial^{2} T_{w}}{\partial x^{2}}+\frac{\partial^{2} T_{w}}{\partial y^{2}}\right)
$$


Introducing the following non-dimensional varibles:

$$
\begin{aligned}
(X, Y) & =\frac{(x, y)}{H},(U, V)=\frac{(u, v)}{\alpha / H} \\
t & =\frac{t^{*}}{H^{2} / \alpha}, P=\frac{\left(p-p_{0}\right)}{\rho_{0}(\alpha / H)^{2}}, \theta=\frac{T-T_{c}}{T_{h}-T_{c}}
\end{aligned}
$$

The non-dimensional form of Equations (6)-(10) are:

$$
\begin{aligned}
& \frac{\partial U}{\partial X}+\frac{\partial V}{\partial Y}=0 \\
& \frac{\partial U}{\partial t}+U \frac{\partial U}{\partial X}+V \frac{\partial U}{\partial Y}=-\frac{\partial P}{\partial X}+\operatorname{Pr} \nabla^{2} U \\
& \frac{\partial V}{\partial t}+U \frac{\partial V}{\partial X}+V \frac{\partial V}{\partial Y}=-\frac{\partial P}{\partial Y}+\operatorname{Pr} \nabla^{2} V \\
& \quad+R a \operatorname{Pr} \theta_{f}-H a^{2} \operatorname{Pr} \mathrm{V} \\
& \frac{\partial \theta_{f}}{\partial t}+U \frac{\partial \theta_{f}}{\partial X}+V \frac{\partial \theta_{f}}{\partial Y}=\left(\frac{\partial^{2} \theta_{f}}{\partial X^{2}}+\frac{\partial^{2} \theta_{f}}{\partial Y^{2}}\right) \\
& \frac{\partial \theta_{w}}{\partial t}=\alpha^{*}\left(\frac{\partial^{2} \theta_{w}}{\partial X^{2}}+\frac{\partial^{2} \theta_{w}}{\partial Y^{2}}\right)
\end{aligned}
$$

where $\alpha^{*}=\alpha_{w} / \alpha_{f}$ is the thermal diffusivity ratio. The effect of the electromagnetic field is introduced into the equations of motion through Hartmann number $H a=B_{0} H(\sigma / \rho \nu)^{1 / 2}$.

The dimensionless form of the governing Equations (11)-(15) in vorticity-stream function form $(\psi$, $\omega)$ can be written as:

- at $t=0 ; \psi=\theta_{w}=\theta_{f}=0$;

- for $t>0$

- Fluid part

$$
\begin{aligned}
& \frac{\partial^{2} \psi}{\partial X^{2}}+\frac{\partial^{2} \psi}{\partial Y^{2}}+\omega=0 \\
& \frac{\partial \omega}{\partial t}+U \frac{\partial \omega}{\partial X}+V \frac{\partial \omega}{\partial Y}=\operatorname{Pr} \nabla^{2} \omega+\operatorname{Ra} \operatorname{Pr} \frac{\partial \theta_{f}}{\partial X} \\
& \quad-H a^{2} \operatorname{Pr} \frac{\partial V}{\partial X} \\
& \frac{\partial \theta_{f}}{\partial t}+U \frac{\partial \theta_{f}}{\partial X}+V \frac{\partial \theta_{f}}{\partial Y}=\left(\frac{\partial^{2} \theta_{f}}{\partial X^{2}}+\frac{\partial^{2} \theta_{f}}{\partial Y^{2}}\right)
\end{aligned}
$$

- Solid part

$$
\frac{\partial \theta_{w}}{\partial t}=\alpha^{*}\left(\frac{\partial^{2} \theta_{w}}{\partial X^{2}}+\frac{\partial^{2} \theta_{w}}{\partial Y^{2}}\right)
$$

with $U=\frac{\partial \psi}{\partial Y}, V=-\frac{\partial \psi}{\partial X}$ and $\omega=\frac{\partial V}{\partial X}-\frac{\partial U}{\partial Y}$.
The boundary conditions are:

$$
\begin{aligned}
\psi(0, Y) & =\psi(L / H, Y)=\psi(X, 0)=\psi(X, 1)=0 \\
\theta_{w}(0, Y) & =1 ; \theta_{f}(L / H, Y)=0 ; \frac{\partial \theta_{w}(X, 0)}{\partial Y}=0 \\
\frac{\partial \theta_{f}(X, 0)}{\partial Y} & =0 ; \frac{\partial \theta_{w}(X, 1)}{\partial Y}=0 ; \frac{\partial \theta_{f}(X, 1)}{\partial Y}=0 \\
\theta_{f}(D, Y) & =\theta_{w}(D, Y) ;\left.\frac{\partial \theta_{f}(D, Y)}{\partial X}\right|_{\text {fluide }} \\
& =\left.K r \frac{\partial \theta_{w}(D, Y)}{\partial X}\right|_{\text {solide }}
\end{aligned}
$$

The local and average Nusselt numbers are defined by:

$$
N u=\left(-\frac{\partial \theta}{\partial X}\right)_{X=D, L / H} ; \quad \overline{N u}=\int_{0}^{1} N u \mathrm{~d} Y
$$

A uniform mesh $(100,82)$ is used in $X$ and $Y$ directions. Numerical solution of the governing equations is obtained using the ADI method [7] and SOR method [8]. The iteration process is terminated under the following conditions:

$$
\begin{gathered}
\sum_{i, j}\left|\psi_{i, j}^{n}-\psi_{i, j}^{n-1}\right| / \sum_{i, j}\left|\psi_{i, j}^{n}\right| \leqslant 10^{-5} \\
\text { For wall side }\left.\overline{N u}\right|_{X=0}=\left.\overline{N u}\right|_{X=D} \\
\text { For fluid side }\left.\overline{N u}\right|_{X=D}=\left.\overline{N u}\right|_{X=L / H}
\end{gathered}
$$

where $D$ is the dimensionless wall thickness $(D=d / H)$.

A good agreement between our results and the previous studies $[1,4]$ is obtained (see Tabs. 1 and 2).

\section{Results and discussions}

\subsection{Effect of Rayleigh number and wall conductivity $(H a=0)$}

In the absence of a magnetic field $(H a=0)$, Table 3 shows the effect of both Rayleigh number and thermal conductivity ratio on fluid motion and thermal field in the enclosure for poorly conducting wall $(K r=0.1)$, equal wall/fluid conductivity $(K r=1)$ and high conducting wall $(K r=10)$. We observe in Table 3 that the maximum values of dimensionless stream function $\psi_{\max }$ in the fluid part are increasing with the increase of both Rayleigh number and conductivity ratio. As a result natural convection is strengthed because of the increase of the effective temperature difference driving the flow.

The rate of heat transfer across the cavity is obtained by evaluating the average Nusselt number at the active side walls. It is clear from Figure $2 \mathrm{a}$ that the average 
Table 1. Comparison of $\overline{N u}$ with Kaminski solution [1], $D=$ 0.2 .

\begin{tabular}{cccc}
\hline$R a$ & $K r$ & $\begin{array}{c}\text { Reference [1] } \\
\text { grid }(40 \times 30)\end{array}$ & $\begin{array}{c}\text { Present study } \\
\text { grid }(100 \times 82)\end{array}$ \\
\hline \multirow{3}{*}{$7 \times 10^{2}$} & 1 & 0.87 & 0.867 \\
& 5 & 1.02 & 1.017 \\
& 10 & 1.04 & 1.040 \\
$7 \times 10^{4}$ & $\infty$ & 1.06 & 1.063 \\
& 1 & 2.08 & 2.084 \\
& 5 & 3.42 & 3.417 \\
& $\infty$ & 3.72 & 3.719 \\
\hline
\end{tabular}

Table 2. Comparison of $\overline{N u}$ with Rudraiah [4] solutions.

\begin{tabular}{ccc}
\hline $\mathrm{Ha}$ & Reference $[4]$ & Present work \\
\hline 0 & 2.518 & 2.534 \\
10 & 2.223 & 2.241 \\
50 & 1.085 & 1.076 \\
100 & 1.011 & 1.006 \\
\hline
\end{tabular}

Table 3. $\psi_{\max }$ for various $R a$ and $K r . D=0.2, H a=0$.

\begin{tabular}{cccc}
\hline & $R a=10^{4}$ & $R a=10^{5}$ & $R a=10^{6}$ \\
\cline { 2 - 4 } & $\psi_{\max }$ & $\psi_{\max }$ & $\psi_{\max }$ \\
\hline$K r=0.1$ & 2.140 & 4.601 & 6.190 \\
$K r=1$ & 3.495 & 6.041 & 8.241 \\
$K r=10$ & 3.948 & 6.712 & 9.896 \\
\hline
\end{tabular}

Nusselt number is more important for high conductive wall. It is increasing with the increase of Rayleigh number for a given $K r$. This means that convection heat transfer becomes more important for both high $R a$ and $K r$. For poor conductive wall $(K r=0.1)$ where the solid part is an insulation material, the average Nusselt number is almost constant and having low values compared with those $(K r=1$ and 10$)$. This is a logical result since reducing the thermal conducting of the wall leads to the increase in thermal resistance of the overall system and therefore reducing $\overline{N u}$. This indicates that most of heat transfer is dominated by conduction mode. In addition for high conduction wall $(K r=10)$ where the solid part is a good conductive wall convection heat transfer is strengthed and the solid layer tends to become an isothermal wall.

Figures $2 \mathrm{~b}$ and $2 \mathrm{c}$ show for $D=0.2$ and $R a=10^{6}$ the effect of thermal conductivity ratio on wall/fluid interface temperature and temperature profile at $Y=0.5$. A comparison is made with the standard enclosure $(D=0.2$, $K r=\infty)$. The temperature difference between the interface and the cold boundary $(\theta=0)$ is small for walls with poor thermal conductivity. It becomes more important with the increase of $K r$, and leads to increase the average Nusselt number. For high values of $K r$ the interface temperature distribution tends to become uniform which is the standard enclosure $(K r=\infty)$. The temperature profile across the wall/ fluid interface is quite non uniform. This non uniformity has a noticeable effect on the flow field and the flow structure is asymmetric.

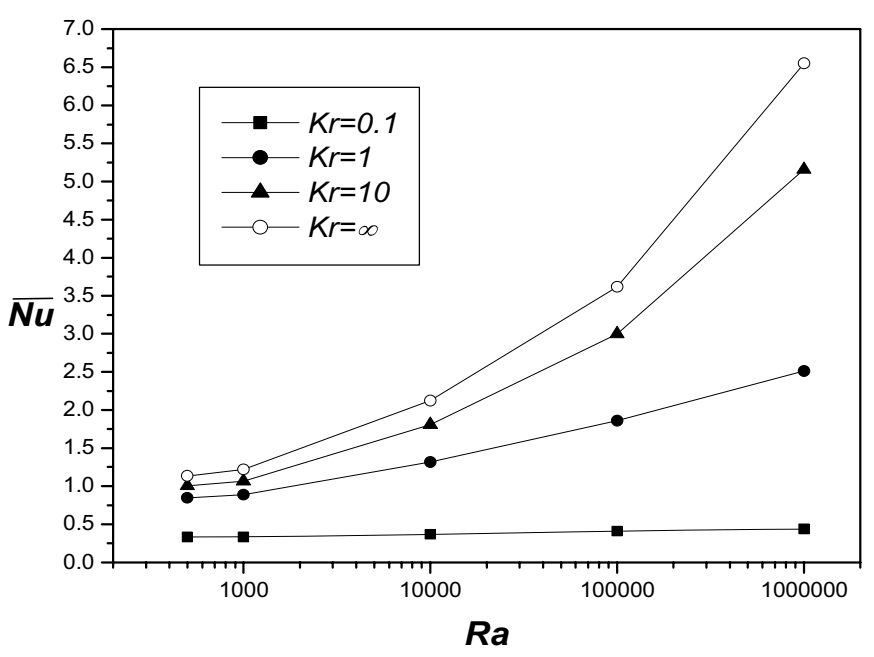

(a)

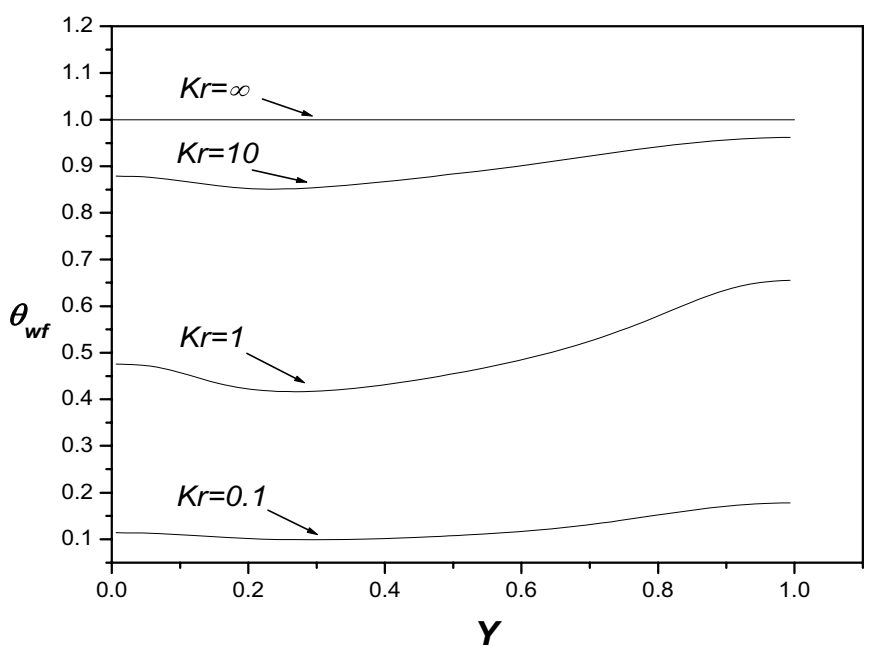

(b)

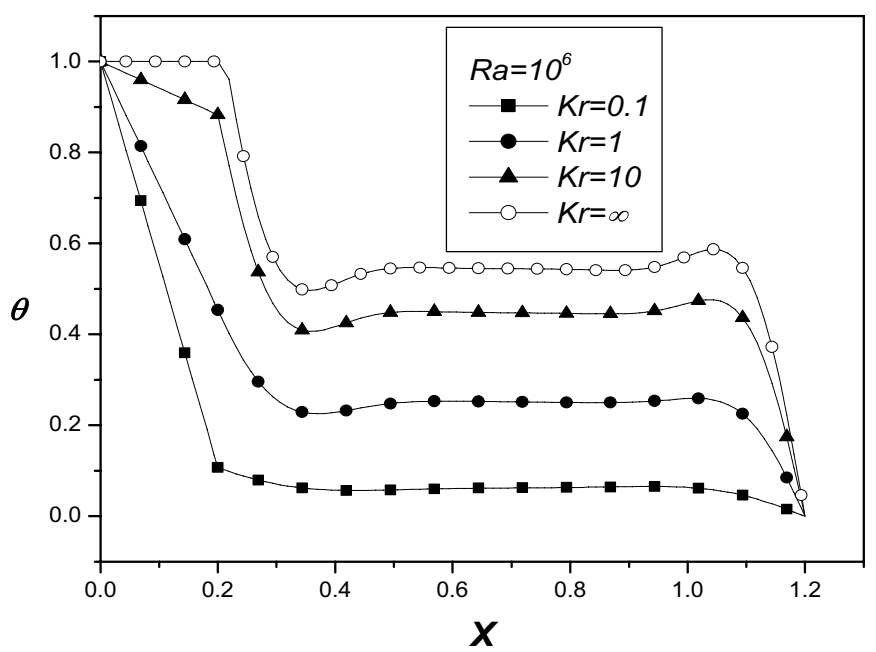

(c)

Fig. 2. (a) Variation of $\overline{N u}$ with Rayleigh number $R a$. (b) Variation of wall/fluid interface temperature $D=0.2$, $R a=10^{6}$. (c) Temperature profile at $Y=0.5$. 
Table 4. $\psi_{\max }$ and $V_{\max }$ for various $H a$ and $K r . D=0.2$.

\begin{tabular}{ccccccc}
\hline & \multicolumn{2}{c}{$H a=50$} & \multicolumn{2}{c}{$H a=100$} & \multicolumn{2}{c}{$H a=150$} \\
\cline { 2 - 7 } & $\psi_{\max }$ & $V_{\max }$ & $\psi_{\max }$ & $V_{\max }$ & $\psi_{\max }$ & $V_{\max }$ \\
\hline$K r=0.1$ & 3.80 & 25.00 & 2.29 & 15.08 & 1.45 & 12.37 \\
$K r=1$ & 6.07 & 55.28 & 4.43 & 34.16 & 3.01 & 21.72 \\
$K r=10$ & 7.41 & 74.37 & 5.66 & 46.21 & 3.82 & 26.75 \\
\hline
\end{tabular}

\subsection{Effect of magnetic field $(\mathrm{Ha} \neq 0)$}

When the fluid is electrically conducting and exposed to a magnetic field the Lorentz force is also active and interacts with buoyancy force in governing the flow and temperature fields. The magnetic field strength and its inclination angle are the most important factors that affect the flow velocity and the rate of heat transfer.

Table 4 demonstrates the influence of the magnetic field parameter $\mathrm{Ha}$ on fluid motion for $\mathrm{Ra}=10^{6} .|\psi|_{\max }$ and $|V|_{\max }$ are reducing by the increase of the magnetic field strength. The flow velocity interacts with the magnetic field and convection heat transfer is considerably reduced. Convection in case $(K r=0.1)$ is weaker than the other cases, so the influence of the magnetic field in this case is less important. For high value of Hartmann number we observe that $|\psi|_{\max }$ and $|V|_{\max }$ are very small comparing with low value of $H a$. This is due to the suppression of convection mechanism by the magnetic field.

The effect of Hartmann number on convection for $R a=10^{6}$, can be observed also from thermal field shown in (Fig. 3). In the absence of a magnetic field $(\mathrm{Ha}=0)$, the fluid rises along the hot wall/fluid surface and falls along the right cold wall, so thermal gradient is very important in these regions. For high $K r\left(R a=10^{6}\right.$, $K r=10$ ), we observe in (Fig. 3c) a temperature stratification in the vertical direction and the thermal boundary layer is well established along the side walls, as a consequence the development of convection mode of heat transfer.

As the Hartmann number increases the stratification of the thermal field ( $K r=1$ and 10) in the interior diminishes and the thermal boundary layers at the two side walls disappear. As shown in (Fig. 3), for poor conductive wall the average Nusselt number is almost constant indicating that the effect of the magnetic field can be neglected since the velocity flow is less important. While for the other cases $(K r=1$ and $K r=10)$ we observe that the rate of heat transfer is decreasing when the magnetic field strength increases and convection is progressively suppressed. In consequence the introduction of a magnetic field is to decrease the overall heat transfer rate between the active walls.

\section{Conclusion}

A numerical study of conjugate natural convection with the presence of a magnetic field was employed to analyze the flow and heat transfer of an electrically conducting fluid (liquid gallium) filled in a square enclosure with (a) $K r=0.1$
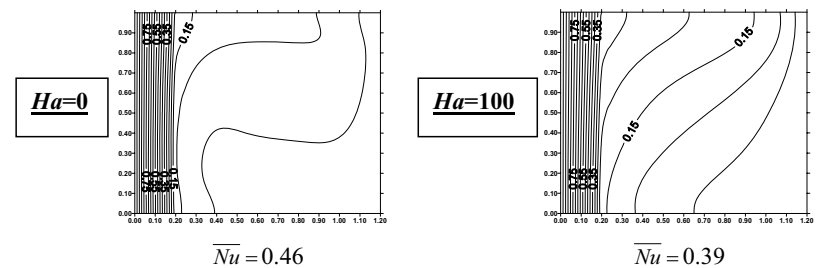

$\overline{N u}=0.39$

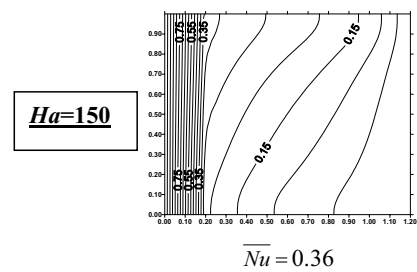

(b) $K r=1$
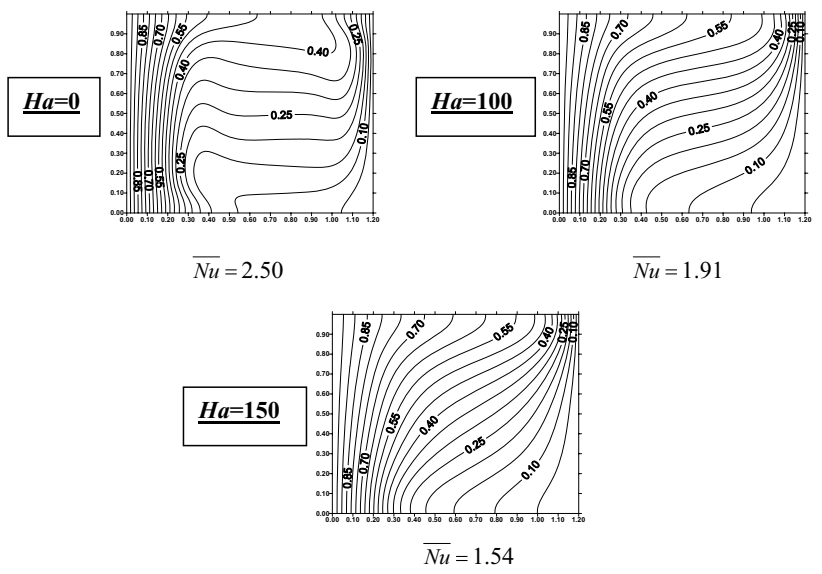

(c) $K r=10$
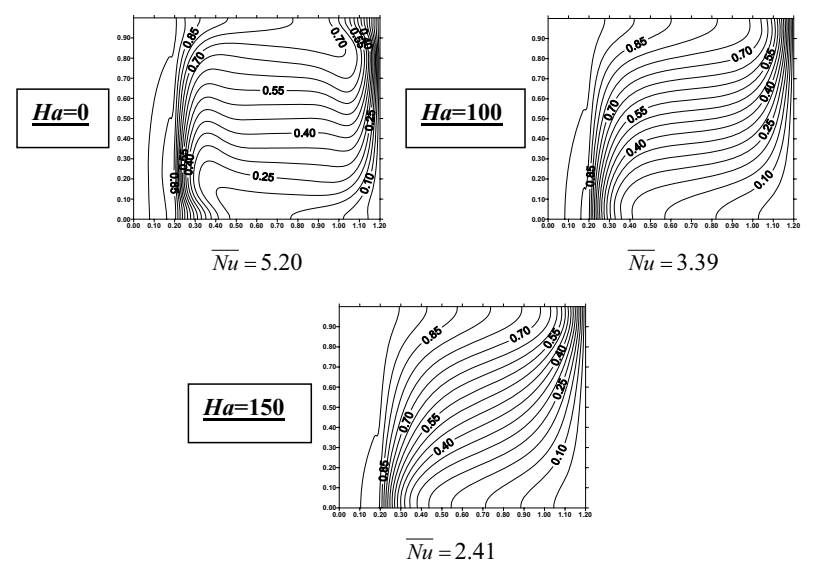

Fig. 3. Steady state of isotherms for different values of Hartmann number.

thick and conductive left vertical wall. The following conclusions were summarized. In the absence of a magnetic field, natural convection is strengthed by the increase of both Rayleigh number and conductivity ratio because of the increase of the effective temperature difference driving the flow. The interface temperature is found to be quite non- uniform especially for high $R a$ and $K r$. This 
non uniformity tends to make the flow pattern in the enclosure asymmetric. The presence of a magnetic field is to control fluid flow and heat transfer. It makes a strong effect on natural convection in the fluid part especially for high conducting wall. The Lorentz force produced by the magnetic field interacts with buoyancy force governing the flow and suppresses the convection currents by reducing the flow velocity and the rate of heat transfer. Furthermore for poor conducting wall $(K r=0.1)$ where the convection is dominated by heat conduction for both wall and fluid layer, results show that the presence of a magnetic field is not important and its effect in this case can be neglected.

\section{References}

[1] D.A. Kaminski, C. Prakash, Conjugate natural convection in a square enclosure: effect of conduction in one of the vertical walls, Int. J. Heat Mass transfer 12 (1986) 1979-1988

[2] D.M. Kim, R. Viskanta, Effect of wall heat conduction on natural convection heat transfer in a square enclosure, J. Heat Transfer 107 (1985) 139-146
[3] A. Liaqat, A.C. Baytas, Conjugate natural convection in a square enclosure containing volumetric sources, Int. J. Heat Mass transfer 44 (2001) 3273-3280

[4] N. Rudraiah, R.M. Barron, M. Venkatachalappa, C.K. Subbaraya, Effect of a magnetic field on free convection in a rectangular enclosure, Int. J. Eng. Sci. 33 (1995) 1075-1084

[5] T. Weier, J. Huller, G. Gerbeth, F.P. Weiss, Lorentz force influence on momentum and mass transfer in natural convection copper electrolysis, Chem. Eng. Sci. 60 (2005) 293-298

[6] M. Pirmohammadi, M. Ghassemi, Effect of magnetic field on convection heat transfer inside a tilted enclosure, Int. Commun. Heat Mass Transfer 36 (2009) 776-780

[7] D.W. Peaceman, H.A. Rachford, The numerical solution of parabolic and elliptic difference equations, J. Soc. Ind. Appl. Math. 3 (1955) 28-43

[8] S.V. Patankar, Numerical Heat Transfer and Fluid Flow, Hemisphere, McGraw-Hill, Washington, DC, 1980

[9] P.J. Roache, Computational fluid dynamics, Hermosa Publishers, 1985 